\title{
Produção de snacks de terceira geração por extrusão de misturas de farinhas de pupunha e mandioca
}

Production of third generation snacks by extrusion-cooking of pupunha and cassava flour mixtures

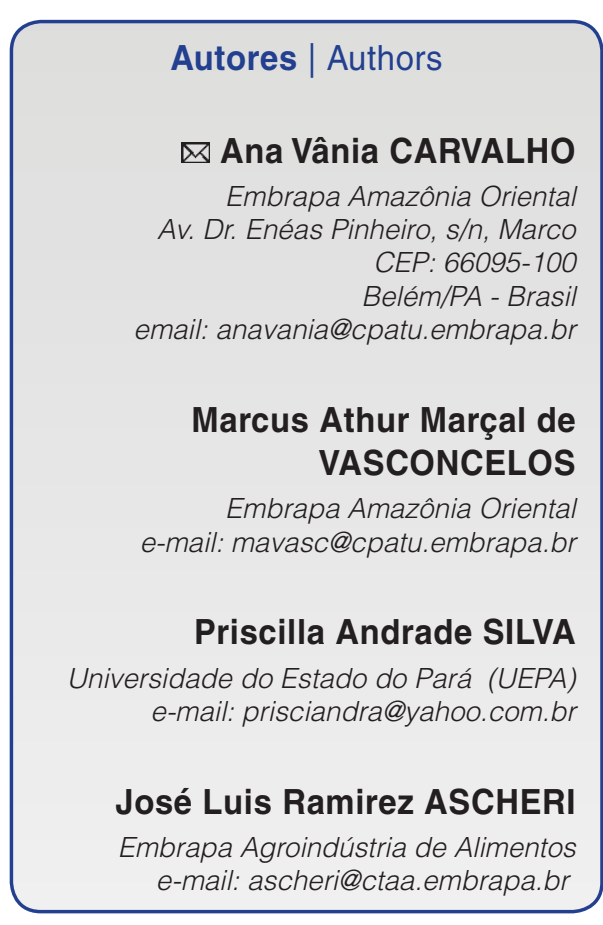

Autor Correspondente / Corresponding Author

Recebido / Received: 27/08/2008 Aprovado / Approved: 23/11/2009

\section{Resumo}

Este trabalho teve por objetivo desenvolver novas formulações de snacks de terceira geração por extrusão termoplástica de misturas de farinhas de pupunha e mandioca e averiguar o potencial do novo produto. A farinha de pupunha foi incorporada à farinha de mandioca nas proporções de 15, 20 e 25\%. Os snacks foram produzidos em extrusora monorrosca, escala piloto, secos a $60^{\circ} \mathrm{C}$ durante $3 \mathrm{~h}$, e expandidos através de fritura em óleo a $180^{\circ} \mathrm{C}$ durante $5-8 \mathrm{~s}$. As amostras de snacks foram submetidas à caracterização físico-química e sensorial. Observou-se o efeito da farinha de pupunha em aumentar os níveis de lipídios, fibras e carotenoides totais nos snacks obtidos. Todas as formulações estudadas foram aceitas sensorialmente, com médias de aceitação de 7,50, 7,15 e 6,73 (em escala hedônica de 9 pontos) em relação à impressão global, para as formulações com 15, 20 e 25\% de farinha de pupunha, respectivamente. Conclui-se que é possível produzir snacks de terceira geração por extrusão a partir da incorporação de 15, 20 ou $25 \%$ de farinha de pupunha à farinha de mandioca, resultando em produtos aceitos sensorialmente e com melhor valor nutricional.

Palavras-chave: Bactris gasipaes; Manihot esculenta;

Extrusados de terceira geração; Aceitabilidade sensorial.

\section{Summary}

The objective of this study was to develop new third generation formulations for snacks by extrusion-cooking using mixtures of pupunha (Bactris gasipaes sp.) and cassava flours, and to verify the potential for this new product. The pupunha flour was incorporated into the cassava flour in proportions of 15, 20 and $25 \%$. The snacks were produced in a single-screw extruder on a pilot scale, dried at $60{ }^{\circ} \mathrm{C}$ for $3 \mathrm{~h}$, and then expanded by frying in oil at $180^{\circ} \mathrm{C}$ for $5-8 \mathrm{~s}$. The extruded samples were submitted to a physicochemical and sensory characterization. The pupunha flour increased the total lipid, fibre and carotenoid contents of the snacks obtained. All the formulations were well accepted, with average overall acceptance scores of 7.50, 7.15 and 6.73 (on a 9-point hedonic scale) for the formulations with 15,20 and $25 \%$ of pupunha flour, respectively. It was concluded that third generation extruded snacks could be produced by incorporating 15,20 or $25 \%$ pupunha flour into the cassava flour matrix, resulting in products accepted by the consumers and with better nutritive value.

Key words: Bactris gasipaes; Manihot esculenta; Third generation extruded products; Consumer acceptance. 


\section{Introdução}

A pupunheira (Bactris gasipaes) é uma palmeira multicaule nativa do clima tropical úmido da região amazônica. Produz frutos comestíveis denominados pupunha de sabor muito apreciado, fazendo parte dos hábitos alimentares da região amazônica (FERREIRA e PENA, 2003). Os frutos, geralmente consumidos após cozimento em água e sal, podem também ser utilizados na fabricação de farinhas para usos variados, representando uma fonte de alimento potencialmente nutritiva, devido ao seu alto conteúdo de carotenoides biodisponíveis, além de teores consideráveis de carboidratos, proteínas e lipídios (YUYAMA et al., 1991; GOIA, 1992; YUYAMA e COZZOLINO, 1996).

O processo de extrusão termoplástica vem ganhando destaque e expansão na indústria de alimentos por ser uma importante técnica que, além de aumentar a variedade de alimentos processados, apresenta muitas vantagens quando comparado a outros sistemas de processamento de alimentos, como versatilidade, custo relativamente baixo, alta produtividade e, por representar um processo ambientalmente seguro, é uma tecnologia catalogada como limpa (CARVALHO, 2000; GUY, 2001).

Durante o processo de extrusão, a matéria-prima é forçada a passar através de um molde em condições de mistura e aquecimento, pressão e fricção que levam à gelatinização do amido e desnaturação de proteínas, dando origem a alimentos como cereais matinais, snacks (expandidos ou não, como no caso dos pellets), alimentos infantis e sopas instantâneas (ARÊAS, 1996; ASCHERI et al., 2000; GUY, 2001).

Os snacks de terceira geração, denominados também de produtos intermediários (half-products) ou pellets, são produtos que não estão expandidos após a extrusão. Estes são submetidos posteriormente às etapas de secagem e de expansão por meio da fritura rápida em óleo, por aquecimento com ar quente ou forno de micro-ondas, estando então prontos para o consumo. Este tipo de produto apresenta baixa umidade (4 a 10\%), permitindo seu armazenamento por longo período de tempo sem deterioração microbiana. Os extrusados de terceira geração são elaborados a partir de farinhas de cereais e tubérculos, amidos, entre outros (RAMÍREZ e WANDERLEI, 1997; ASCHERI et al., 2000; CARVALHO et al., 2002).

O objetivo deste trabalho foi desenvolver novas formulações de snacks por extrusão termoplástica utilizando misturas de farinhas de pupunha e mandioca e avaliar o efeito da formulação nas características físicoquímicas e sensoriais do produto final.

\section{Material e métodos}

\subsection{Matéria-prima}

Foram utilizados farinha de mandioca, adquirida em feiras livres na cidade de Belém, Pará, e frutos de pupunheira, obtidos de cultivos comerciais no município de Santa Isabel, Pará, os quais foram processados para obtenção da farinha. No preparo da farinha, as pupunhas foram lavadas em solução de hipoclorito de sódio a $100 \mathrm{mg} \cdot \mathrm{L}^{-1}$ por $15 \mathrm{~min}$, descascadas, as sementes retiradas e, a seguir, submetidas ao cozimento em água fervente durante 20 min. Após secagem em estufa com circulação forçada de ar a $60^{\circ} \mathrm{C}$, e umidade entre 7 e $8 \%$, foram trituradas em moinho de facas tipo Willye (TE-650 - Tecnal, Piracicaba, SP) até tamanho de partículas entre 9 e 20 mesh e embaladas em sacos de polietileno até o momento da utilização.

\subsection{Processamento dos snacks}

As formulações testadas foram preparadas por adição de farinha de pupunha à farinha de mandioca nas proporções de 15, 20 e 25\%. Nas três formulações, adicionou-se $1,5 \%$ de sal, sendo as amostras condicionadas para o teor de umidade de $35 \%$. Visando avaliar o comportamento do ácido fólico perante o processamento por extrusão, as formulações estudadas foram enriquecidas com $28 \mathrm{mg} .100 \mathrm{~g}^{-1}$ do premix de vitaminas e minerais da Fortitech (Brasil), incorporando-se, deste modo, $200 \mu \mathrm{g}$ de ácido fólico para cada $100 \mathrm{~g}$ de farinha mista. Definiu-se a quantidade do premix a ser adicionada em função da quantidade de ácido fólico recomendada na Resolução RDC n 344 de 13 de dezembro de 2002, que trata do Regulamento Técnico para a Fortificação das Farinhas de Trigo e das Farinhas de Milho com Ferro e Ácido Fólico. Segundo a Resolução, cada 100 g de farinha de trigo e de farinha de milho devem fornecer no mínimo 150 mcg (cento e cinquenta microgramas) de ácido fólico.

As diferentes formulações testadas foram processadas em extrusora monorrosca (Labor PQ30 - INBRAMAQ, Indústria de Máquinas Ltda., Ribeirão Preto, São Paulo), de configuração e parafuso intercambiáveis, apropriada para produção de produtos não expandidos em canhão de maior comprimento, com controle de temperatura nas diferentes zonas de aquecimento e velocidade do parafuso regulável, através de regulador e frequência. Foram adotados os seguintes parâmetros: temperatura nas zonas do extrusor (Zona $1=30{ }^{\circ} \mathrm{C}$; Zona $2=40{ }^{\circ} \mathrm{C}$; Zona $3=60{ }^{\circ} \mathrm{C}$; Zona $4=65^{\circ} \mathrm{C}$; Zona $\left.5=70{ }^{\circ} \mathrm{C}\right)$, velocidade do parafuso (177 rpm), taxa de alimentação de 292 g. $\mathrm{min}^{-1}$ e matriz laminar de $1 \mathrm{~mm}$. A temperatura de secagem após o processo de extrusão foi de $60^{\circ} \mathrm{C}$ em estufa com circulação de ar, durante 3 h. Os extrusados 
secos foram fritos em óleo à temperatura de $180^{\circ} \mathrm{C}$ por cerca de 5 a 8 s, sendo mantidos em embalagem flexível laminada (BOPPmetalizado/PE/BOPP) em temperatura ambiente, até o momento das análises (realizadas em um prazo de 15 dias).

\subsection{Caracterização físico-química das matérias-primas e dos extrusados}

As farinhas de pupunha e mandioca bem como os snacks obtidos foram caracterizados quanto à atividade de água por medição direta em analisador de atividade de água marca DECAGON, modelo Pawkit (Pullman, EUA), teores de umidade (método gravimétrico $n^{\circ}$ 920.151), cinzas (método gravimétrico n 940.26), proteínas (método de Kjeldahl, $\mathrm{n}^{\circ}$ 920.152), lipídios (método gravimétrico $n^{\circ}$ 920.39) e fibra alimentar total, solúvel e insolúvel (método enzimático-gravimétrico $n^{\circ}$ 991.43) (HORWITZ, 1997). As farinhas de pupunha e mandioca também foram avaliadas quanto ao pH (HORWITZ, 1997) e amido (RICKARD e BEHN, 1987). Foi determinado o teor de carotenoides totais da farinha de pupunha e dos produtos obtidos, segundo metodologia descrita por Godoy e Rodriguez-Amaya (1994), sendo realizada etapa de saponificação somente para os snacks, de acordo com Sentanin e Rodriguez-Amaya (2007). Os snacks foram analisados ainda quanto ao teor de ácido fólico (CATHARINO et al., 2003), carboidratos (calculado pela diferença entre 100 e a soma das porcentagens de água, proteína, lipídios e cinzas) e valor energético total (USDA, 1963). Todas as análises foram realizadas em triplicata, à exceção da análise de ácido fólico que foi realizada em duplicata. $\mathrm{O}$ valor energético total foi calculado através da seguinte Equação 1:

$\operatorname{VET}=(C \times 4)+(A \times 4)+(B \times 9)$

onde C: carboidratos (descontado do teor de fibra total), A: proteína total e B: extrato etéreo.

\subsection{Análise microbiológica dos snacks}

Realizou-se análise de coliformes a $45^{\circ} \mathrm{C}$, utilizando-se o meio de cultura Lauryl Sulfato Triptose (LST) para a inoculação de coliformes em série de 3 tubos, contendo tubo de Durhan invertido, sendo aplicado o método do número mais provável (NMP) por grama de amostra, de acordo com Vanderzant e Splittstoesser (1992).

\subsection{Análise sensorial}

As formulações foram avaliadas quanto à aceitação, através de escala hedônica estruturada de nove pontos ( 9 = gostei muitíssimo; 1 = desgostei muitíssimo) e intenção de compra, escala estruturada de cinco pontos $(1=$ certamente não compraria; 5 = certa- mente compraria) (STONE e SIDEL, 1993). A avaliação sensorial foi realizada por 40 provadores não treinados, entre funcionários, visitantes e estagiários da Embrapa Amazônia Oriental. As três amostras de snacks foram apresentadas monadicamente aos potenciais consumidores à temperatura ambiente, em pratos plásticos descartáveis codificados com números aleatórios de três dígitos e avaliadas quanto à textura, sabor, impressão global e intenção de compra. As amostras foram servidas nos pratos somente no momento da análise para evitar absorção de umidade, sendo oferecidas cerca de $5 \mathrm{~g}$ de cada amostra por provador.

\subsection{Análise estatística}

Os resultados das características avaliadas foram submetidos à análise de variância e as médias, quando significativas, comparadas pelo Teste de Tukey a 5\% de probabilidade, utilizando-se o software SAS ${ }^{\circledR}$ versão 8.0 (SAS, 1999).

\section{Resultados e discussão}

\subsection{Caracterização físico-química das farinhas de pupunha e mandioca}

Observam-se, na Tabela 1, os valores médios da caracterização físico-química das matérias-primas utilizadas na formulação dos extrusados.

Os valores encontrados para a caracterização da farinha de pupunha (Tabela 1) estão próximos aos relatados na literatura consultada (OLIVEIRA et al., 2007; ANDRADE et al., 2003; CLEMENT et al., 1998): teor de proteínas varia de 2,41 a 7,10\%; lipídios, de 8,8 a 21,00\%; cinzas, de 1,33 a 2,2\%; e teor de amido de $82,07 \%$. 0 teor de fibra alimentar total observado neste trabalho $(10,52 \%)$ está próximo ao encontrado por Oliveira et al. (2007), 8,80\%, que também analisou a farinha de pupunha com casca. O valor de carotenoides totais obtido para a farinha de pupunha $\left(137,98 \mu \mathrm{g} \cdot \mathrm{g}^{-1}\right)$ está superior ao relatado por Andrade et al. (2003), que encontrou teores de 45,56 $\mu \mathrm{g} \cdot \mathrm{g}^{-1}$ na polpa de pupunha in natura e $103,81 \mu \mathrm{g} \cdot \mathrm{g}^{-1}$ na pupunha cozida, ambas em base seca. Deve-se ressaltar que no presente trabalho obteve-se a farinha de pupunha a partir de frutos com casca e, também, utilizaram-se dois processamentos térmicos no preparo da farinha (cozimento e posterior secagem em estufa) o que pode ter contribuído para o maior teor de carotenoides totais observados neste trabalho. Segundo Andrade et al. (2003), ocorrem mudanças químicas nesses pigmentos por ocasião do cozimento, provavelmente descomplexação de carotenoides, o que reflete na intensificação da coloração alaranjada e aumento do teor de carotenoides totais na pupunha cozida. As diferenças observadas entre os trabalhos provavelmente sejam devidas às diferentes linhagens e variedades (raças) de 
Tabela 1. Caracterização físico-química de farinhas de pupunha e mandioca.

\begin{tabular}{lrr}
\multicolumn{1}{c}{ Determinação } & \multicolumn{1}{c}{$\begin{array}{c}\text { Farinha de } \\
\text { pupunha }\end{array}$} & $\begin{array}{c}\text { Farinha de } \\
\text { mandioca }\end{array}$ \\
\hline Atividade de água & $0,51 \pm 0,01$ & $0,52 \pm 0,01$ \\
$\mathrm{pH}$ & $6,16 \pm 0,01$ & $4,38 \pm 0,01$ \\
Umidade (\%) & $6,21 \pm 0,24$ & $9,09 \pm 0,10$ \\
Proteína (\%)* & $4,15 \pm 0,04$ & $0,93 \pm 0,01$ \\
Lipídios $(\%)^{*}$ & $11,56 \pm 0,25$ & $0,13 \pm 0,01$ \\
Cinzas $(\%)^{*}$ & $1,53 \pm 0,03$ & $0,86 \pm 0,05$ \\
Fibra total $(\%)^{*}$ & $10,52 \pm 0,05$ & $4,75 \pm 0,02$ \\
Fibra insolúvel $(\%)^{*}$ & $8,62 \pm 0,02$ & $2,94 \pm 0,01$ \\
Fibra solúvel $(\%)^{*}$ & $1,90 \pm 0,02$ & $1,82 \pm 0,02$ \\
Amido (\%) & $70,54 \pm 0,03$ & $88,34 \pm 0,02$ \\
Carotenoides totais $\left(\mu \mathrm{g} \cdot \mathrm{g}^{-1}\right)$ & $137,98 \pm 2,45$ & - \\
\hline
\end{tabular}

${ }^{*}$ Resultados em base seca.

pupunhas estudadas, além da forma de processamento dos frutos.

Os resultados encontrados para a farinha de mandioca também estão de acordo com a literatura consultada (BRASIL, 1995; CHISTÉ et al., 2006; CHISTÉ et al., 2007; SOUZA et al., 2008), que relata teores variando de 0,38 a 0,93\% de cinzas; 0,11 a 1,91\% de lipídios; e 0,53 a 2,58\% de proteínas. Para o teor de fibras, o valor encontrado neste trabalho está superior aos relatos por Souza et al. (2008), variando de 1,60\% a $2,71 \%$, provavelmente devido aos métodos de análise diferentes utilizados nos dois trabalhos. Observou-se em relação aos valores de umidade, cinzas e amido que a amostra analisada está de acordo com os padrões estabelecidos pela legislação brasileira para farinhas de mandioca, Portaria n 554 de 30 de agosto de 1995 do Ministério da Agricultura, do Abastecimento e Reforma
Agrária (BRASIL, 1995), que estabelece valores máximos de $13 \%$ de umidade, $1,5 \%$ de cinzas e o mínimo de $70 \%$ de amido para farinhas do grupo seca.

\subsection{Caracterização físico-química dos snacks}

A caracterização físico-química dos snacks de pupunha e mandioca é apresentada na Tabela 2.

De maneira geral, observam-se diferenças nas características físico-químicas entre as diferentes formulações, para as análises de proteínas, lipídios, fibra alimentar e carotenoides totais, verificando-se aumento no teor desses constituintes com a maior porcentagem de farinha de pupunha incorporada à farinha de mandioca.

Os valores de umidade encontrados neste trabalho, de 5,45 a 6,06\%, estão próximos aos relatados em trabalho realizado por Carvalho (2000) com snacks de terceira geração obtidos de misturas de trigo, arroz e banana, com teores de umidade variando de 7,73 a 10,75\% em função da formulação empregada. Com relação à análise de lipídios, antes do processo de fritura, observam-se teores variando de 1,16, 1,24 e 2,19\% de lipídios para as formulações de extrusados com 15, 20 e $25 \%$ de farinha de pupunha, respectivamente. Assim, de acordo com a Tabela 2, verificam-se altos teores de lipídios nos extrusados fritos, com a incorporação média de $89 \%$ de óleo durante o processo de fritura.

Os valores de atividade de água, variando de 0,23 a 0,24 , para as três formulações de extrusados fritos, estão próximos aos observados por Souza (2003), estudando cereais matinais extrusados de castanha-do-brasil com mandioca, que relata atividade de água igual a 0,18.

Embora o teor de umidade seja um parâmetro importante na conservação dos alimentos, em muitos casos a atividade de água tem sido o parâmetro prefe-

Tabela 2. Caracterização físico-química dos snacks de farinha de pupunha e mandioca, em base úmida.

\begin{tabular}{|c|c|c|c|}
\hline \multirow[t]{2}{*}{ Determinação } & \multicolumn{3}{|c|}{ Extrusados Fritos } \\
\hline & $15 \% *$ & $20 \% *$ & $25 \%$ * \\
\hline Atividade de água & $0,24 \pm 0,00^{a}$ & $0,24 \pm 0,00^{\mathrm{ab}}$ & $0,23 \pm 0,01^{b}$ \\
\hline Umidade (\%) & $5,83 \pm 0,42^{a}$ & $5,45 \pm 0,29^{a}$ & $6,06 \pm 0,20^{a}$ \\
\hline Proteína (\%) & $1,20 \pm 0,04^{b}$ & $1,26 \pm 0,09^{a}$ & $1,28 \pm 0,05^{a}$ \\
\hline Lipídios (\%) & $13,06 \pm 0,06^{b}$ & $13,49 \pm 0,29^{b}$ & $14,65 \pm 0,74^{a}$ \\
\hline Cinzas (\%) & $1,15 \pm 0,16^{a}$ & $1,37 \pm 0,10^{a}$ & $1,35 \pm 0,15^{a}$ \\
\hline Fibra total (\%) & $4,93 \pm 0,07^{c}$ & $5,67 \pm 0,14^{b}$ & $6,22 \pm 0,09^{a}$ \\
\hline Fibra insolúvel (\%) & $2,97 \pm 0,09^{b}$ & $3,17 \pm 0,16^{\mathrm{ab}}$ & $3,47 \pm 0,09^{a}$ \\
\hline Fibra solúvel (\%) & $1,97 \pm 0,10^{b}$ & $2,50 \pm 0,20^{a}$ & $2,75 \pm 0,17^{a}$ \\
\hline Carboidratos (\%) & $78,76 \pm 0,17^{\mathrm{a}}$ & $78,43 \pm 0,20^{a}$ & $76,66 \pm 0,31^{b}$ \\
\hline Valor energético (Kcal.100 g ${ }^{-1}$ ) & $417,66 \pm 0,14^{a}$ & $417,49 \pm 0,19^{a}$ & $418,73 \pm 0,32^{a}$ \\
\hline Ácido fólico $\left(\mu \mathrm{g} .100 \mathrm{~g}^{-1}\right)$ & $63 \pm 15^{a}$ & $81 \pm 14^{a}$ & $72 \pm 21^{a}$ \\
\hline Carotenoides totais $\left(\mu \mathrm{g} \cdot \mathrm{g}^{-1}\right)$ & $1,27 \pm 0,01^{c}$ & $2,58 \pm 0,33^{b}$ & $3,49 \pm 0,24^{a}$ \\
\hline
\end{tabular}

Médias com letras iguais, em uma mesma linha, não diferem entre si pelo Teste de Tukey, a $5 \%$ de probabilidade; e * Porcentagem de farinha de pupunha incorporada à farinha de mandioca. 
rido para ser medido e acompanhado, por representar melhor a água disponível ou o estado da água disponível que melhor se correlaciona com a conservação dos alimentos (CHIRIFE e BUERA, 1995). Com atividade de água de 0,23 e 0,24, observadas neste trabalho, podemos classificar os extrusados fritos como alimentos de baixa atividade de água. Os alimentos com teor baixo de água apresentam níveis de umidade inferiores a 20\% e atividade de água abaixo de 0,60, sendo, por isto, microbiologicamente estáveis, desde que não haja absorção de umidade durante a estocagem (UBOLDI-EIROA, 1981; MORITA et al., 2005).

Os carotenoides, além de serem corantes naturais dos alimentos, possuem atividades biológicas, sendo considerados compostos bioativos que proporcionam benefícios adicionais à saúde (SENTANIN e RODRIGUEZ-AMAYA, 2007). Porém, são compostos muito sensíveis à luz, oxigênio e temperatura elevada, sendo degradados facilmente quando expostos a estas condições. Para a análise de carotenoides totais, observam-se perdas consideráveis em função das etapas de extrusão e fritura, comprovando a sensibilidade dos carotenoides frente ao processamento. Porém, mesmo devido às perdas ocorridas durante o processamento, os valores encontrados para os extrusados fritos, variando de 1,27 a 3,49 $\mu \mathrm{g} \cdot \mathrm{g}^{-1}$ em função da formulação, são consideráveis quando comparados aos teores observados para algumas frutas in natura, como pêssego $\left(5,2 \mu \mathrm{g} \cdot \mathrm{g}^{-1}\right)$, nectarina $\left(5,5 \mu \mathrm{g} \cdot \mathrm{g}^{-1}\right)$ e maracujá $\left(4,7 \mu \mathrm{g} \cdot \mathrm{g}^{-1}\right)$ (GODOY e RODRIGUEZ-AMAYA, 1994).

Os snacks obtidos a partir de farinha de pupunha e mandioca apresentam teores de fibra alimentar variando de 4,93 a 6,22 g. $100 \mathrm{~g}^{-1}$ do produto pronto para o consumo. Desta forma, os produtos com incorporação de 15\% e 20\% de farinha de pupunha podem receber a alegação de alimento fonte de fibras (>3 g.100 g $\mathrm{g}^{-1}$ ), enquanto que o produto com $25 \%$ de farinha de pupunha pode receber a alegação de alto teor de fibras (>6 g.100 g-1), de acordo com a Portaria $n^{\circ} 27$ de 13 de janeiro de 1998, do Ministério da Saúde. Verifica-se ainda que os teores de fibras observados neste trabalho são superiores aos relatados por Menezes et al. (2001), para alguns cereais matinais, como cereal matinal de aveia, amêndoa e mel (4,90\%), cereal matinal de milho Corn Flakes $\AA(3,57 \%)$ e cereal matinal de milho, trigo e aveia (2,15\%), e por Carvalho (2000), estudando snacks de terceira geração obtidos a partir de farinhas de banana e arroz, com teores variando de 4,26 a 4,98\%, em função da formulação testada.

Os resultados de carboidratos e valor energético dos snacks (Tabela 2) mostram desempenho de um produto energético, podendo ser utilizado na alimentação humana para suprir carência de alimentos energéticos. Além disso, o enriquecimento dos extrusados com ácido fólico pode representar interessante complemento na dieta desta importante vitamina. Observam-se teores razoáveis de ácido fólico nos extrusados fritos, entre 63 e $81 \mu \mathrm{g} .100 \mathrm{~g}^{-1}$, correspondendo entre 32 a $41 \%$ da ingestão diária recomendada desta vitamina para adultos, sendo considerado, de acordo com a Portaria $n^{\circ} 31$ de 31 de janeiro de 1998 da ANVISA, um alimento rico em ácido fólico (BRASIL, 1998b; BRASIL, 1998c). Observase ainda que, embora o teor de ácido fólico encontrado nos snacks os enquadre como alimentos ricos em ácido fólico, houve perda desta vitamina por ocasião do processamento, variando de 58 a 69\% do total incorporado. No organismo humano o ácido fólico tem papel essencial no metabolismo dos aminoácidos, na síntese de ácidos nucleicos, na formação de células do sangue e de alguns constituintes do tecido nervoso. A deficiência severa geralmente provoca anemia megaloblástica que, se não tratada, pode levar à morte. A vitamina previne ainda a má formação do tubo neural (estrutura precursora do cérebro e da medula espinhal) no feto (GERMANI e SILVA, 2004; ANVISA, 2007).

\subsection{Análise microbiológica}

A ANVISA, através da Resolução n 12, de 2 de janeiro de 2001, regulamenta os padrões microbiológicos para alimentos e estabelece, para cereais matinais e produtos extrusados, valores inferiores a 1 UFC. $g^{-1}$ para coliformes a $45^{\circ} \mathrm{C}$. Para as três amostras de extrusados, 15, 20 e $25 \%$ de farinha de pupunha, os valores encontrados foram inferiores ao estabelecido na legislação citada, estando as três formulações de extrusados aptas para serem consumidas, indicando boas condições higiênicas durante todo o processamento dos produtos.

\subsection{Análise sensorial}

Na Tabela 3 estão apresentadas as médias do teste de aceitação e intenção de compra, realizados para a avaliação dos extrusados de pupunha e mandioca.

Os snacks obtidos através da incorporação de farinha de pupunha à farinha de mandioca, nas proporções de 15, 20 e 25\%, foram aceitos sensorialmente, com notas médias variando de 6,70 a 7,50 , valores que

Tabela 3. Aceitabilidade sensorial e intenção de compra dos snacks de farinha de pupunha e mandioca.

\begin{tabular}{lccc}
\multicolumn{1}{c}{ Atributos } & \multicolumn{3}{c}{ Extrusados fritos } \\
& $\mathbf{1 5 \%}^{*}$ & $\mathbf{2 0 \%}^{*}$ & $\mathbf{2 5 \%}^{*}$ \\
\hline Textura & $7,38 \pm 1,27^{\mathrm{a}}$ & $7,28 \pm 1,60^{\mathrm{a}}$ & $6,75 \pm 1,55^{\mathrm{a}}$ \\
Sabor & $7,50 \pm 1,15^{\mathrm{a}}$ & $7,08 \pm 1,40^{\mathrm{a}}$ & $6,70 \pm 1,57^{\mathrm{b}}$ \\
Impressão global & $7,50 \pm 1,09^{\mathrm{a}}$ & $7,15 \pm 1,56^{\mathrm{ab}}$ & $6,73 \pm 1,77^{\mathrm{b}}$ \\
Intenção de & $4,30 \pm 0,79^{\mathrm{a}}$ & $3,90 \pm 1,13^{\mathrm{ab}}$ & $3,68 \pm 1,18^{\mathrm{b}}$ \\
compra & & & \\
\hline
\end{tabular}

Médias com letras iguais, em uma mesma linha, não diferem entre si pelo Teste de Tukey, a $5 \%$ de probabilidade; e * Porcentagem de farinha de pupunha incorporada à farinha de mandioca. 
correspondem aos intervalos de notas de 6 a 8 e que representam, respectivamente, "gostei ligeiramente" e "gostei muito".

Para o atributo textura, não houve diferença estatística entre as três amostras avaliadas. Já para o sabor, as formulações com $15 \%$ e $20 \%$ de pupunha foram as mais aceitas, não diferindo entre si. Para a intenção de compra, verifica-se que a formulação com 15\% apresentou a maior média em relação às demais, porém não apresentou diferença estatisticamente significativa com a formulação de $20 \%$ de pupunha. O mesmo foi verificado em relação à impressão global.

Os resultados do teste de intenção de compra dos snacks de pupunha e mandioca podem ser visualizados na Figura 1.

Como se pode observar, o nível de rejeição das formulações de extrusados foi muito baixo, sendo que, de 40 provadores, apenas dois citaram que certamente não comprariam a formulação com 20\% e 4 provadores certamente não comprariam a formulação com $25 \%$ de pupunha.

Além disso, para as três formulações estudadas, entre 65 e $85 \%$ dos provadores, opinaram com notas nos níveis 4 ou 5, ou seja, "possivelmente" e "certamente comprariam o produto", o que comprova a boa aceitabilidade dos snacks.

Ainda, através do histograma da Figura 1, pode-se observar que a formulação com 15\% de pupunha se destacou no nível de certeza dos provadores, com

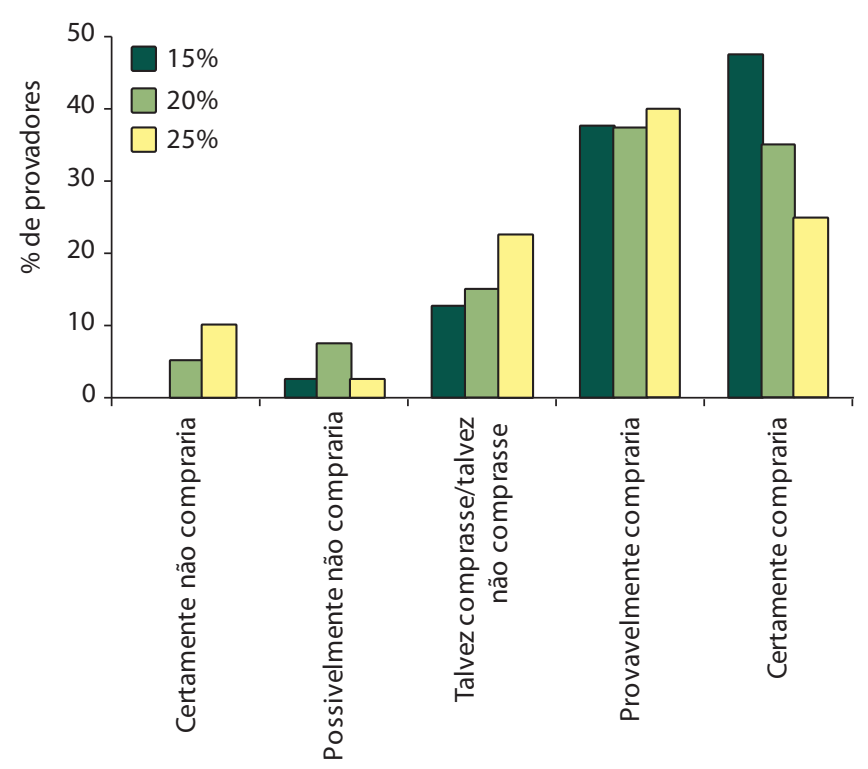

Figura 1. Distribuição percentual da intenção de compra dos snacks.
$85 \%$ dos provadores confirmando que, se esse tipo de produto estivesse à venda, possivelmente ou certamente o comprariam.

\section{Conclusões}

É possível produzir snacks de terceira geração por extrusão a partir da incorporação de 15, 20 ou 25\% de farinha de pupunha à farinha de mandioca. Todas as formulações testadas foram aceitas sensorialmente.

A incorporação de farinha de pupunha ocasiona aumento dos teores de proteínas, lipídios, fibra alimentar e carotenoides totais nos snacks estudados.

\section{Agradecimentos}

Os autores agradecem à Agência de Desenvolvimento da Amazônia (ADA) e Secretaria de Estado de Ciência, Tecnologia e Meio Ambiente do Estado do Pará (SECTAM) o apoio financeiro; e ao Conselho Nacional de Desenvolvimento Científico e Tecnológico (CNPq) a concessão da bolsa de Desenvolvimento Científico Regional para o primeiro autor.

\section{Referências}

ANDRADE, J. S.; PANTOJA, L.; MAEDA, R. N. Melhoria do rendimento e do processo de obtenção da bebida alcoólica de pupunha (Bactris gasipaes Kunth). Ciência e Tecnologia de Alimentos, Campinas, v. 23, n. supl., p. 34-38, 2003.

AGÊNCIA NACIONAL DE VIGILÂNCIA SANITÁRIA - ANVISA. Farinha terá ácido fólico para combater anencefalia em bebês. Brasília: ANVISA, 2007. Disponível em: <http://www. anvisa.gov.br/>. Acesso em: Novembro 2007.

ARÊAS, J. A. G. Interações moleculares do amido durante o processo de extrusão. Boletim da SBCTA, Campinas, v. 30, n. 1, p. 28-30, 1996.

ASCHERI, J. L. R.; CARVALHO, C. W. P.; MATSUURA, F. C. A. $U$. Elaboração de pellets de harina de raspa de mandioca por extrusión termoplástica (escala piloto e industrial). Alimentaria, Madri, v. 37, n. 309, p. 101-106, 2000.

BRASIL. Portaria n. 554 de 30 de Agosto de 1995. Norma de identidade, qualidade, apresentação, embalagem, armazenamento e transporte da farinha de mandioca. Diário Oficial [da] República Federativa do Brasil, Poder Executivo, Brasília, DF, 01 de Setembro de 1995, Seção 1.

BRASIL. Agência Nacional de Vigilância Sanitária. Portaria n. 33 de 13 de Janeiro de 1998a. Adota valores como níveis de IDR para as vitaminas, minerais e proteínas. Diário Oficial da União, Poder Executivo, Brasília, DF, 16 de Janeiro de 1998. Disponível em: <http://www.anvisa.gov.br/legis/portarias/33_98. htm>. Acesso em: Dezembro 2007.

BRASIL. Agência Nacional de Vigilância Sanitária. Portaria n. 27 de 13 de Janeiro de 1998b. Aprova o Regulamento Técnico referente à 
Produção de snacks de terceira geração por extrusão de misturas de farinhas de pupunha e mandioca CARVALHO, A. V. et al.

Informação Nutricional Complementar (declarações relacionadas ao conteúdo de nutrientes), constantes do anexo desta Portaria. Diário Oficial da União, Poder Executivo, Brasília, DF, 16 de Janeiro de 1998. Disponível em: <http://e-legis.anvisa.gov.br/ leisref/public/showAct.php?id=97>. Acesso em: Abril 2008.

BRASIL. Agência Nacional de Vigilância Sanitária. Portaria n. 31 de 31 de Janeiro de 1998c. Aprova o regulamento técnico referente a alimentos adicionados de nutrientes essenciais, constante do anexo desta Portaria. Diário Oficial da União, Poder Executivo, Brasília, DF, 16 de Janeiro de 1998. Disponível em: <http://e-legis.bvs.br/leisref/public/showAct.php>. Acesso em: Abril 2009.

BRASIL. Agência Nacional de Vigilância Sanitária. Resolução RDC n. 12 de 02 de Janeiro de 2001. Aprova o Regulamento Técnico sobre padrões microbiológicos para alimentos. Diário Oficial da União, Poder Executivo, Brasília, DF, 10 de Janeiro de 2001. Disponível em: <http://e-legis.anvisa.gov.br/leisref/public/ showAct. php?id=144>. Acesso em: Dezembro 2007

CARVALHO, R. V. Formulações de snacks de terceira geração por extrusão: caracterização texturométrica e microestrutural. Lavras, 2000. 89 p. Dissertação (Mestrado em Ciência de Alimentos) - Universidade Federal de Lavras - UFLA.

CARVALHO, R. V.; ASCHERI, J. L. R.; CAL-VIDAL, J. Efeito dos parâmetros de extrusão nas propriedades físicas de pellets de misturas de farinhas de trigo, arroz e banana. Ciência e Agrotecnologia, Lavras, v. 26, n. 5, p. 1006-1018, 2002.

CATHARINO, R. R.; VISENTAINER, J. V.; GODOY, H. T. Avaliação das condições experimentais de CLAE na determinação de ácido fólico em leites enriquecidos. Ciência e Tecnologia de Alimentos, Campinas, v. 23, n. 3, p. 389-395, 2003.

CHIRIFE, J.; BUERA, M. P. A critical review of some non-equilibrium situations and glass transitions on water activity values of foods in the microbiological growth range. Journal of Food Engineering, Barking, v. 25, p. 531-552, 1995.

CHISTÉ, R. C.; COHEN, K. O.; MATHIAS, E. A.; RAMOA Jr., A. G. A. Estudo das propriedades físico-químicas e microbiológicas no processamento da farinha de mandioca do grupo d'água. Ciência e Tecnologia de Alimentos, Campinas, v. 27, n. 2, 2007. Disponível em: <http://www.scielo.br/scielo.php?script=sci_ arttext\&pid=S0101-20612007000200009\&Ing=en\&nrm=iso $>$. Acesso em: Agosto 2008.

CHISTÉ, R. C.; COHEN, K. O.; MATHIAS, E. A.; RAMOA Jr., A. G. A. Qualidade da farinha de mandioca do grupo seca. Ciência e Tecnologia de Alimentos, Campinas, v. 26, n. 4, 2006. Disponível em: <http://www.scielo.br/scielo.php?script=sci_ arttext\&pid=S0101-20612006000400023\&lng=en\&nrm=iso> Acesso em: Agosto 2008.

CLEMENT, C. R.; AGUIAR, J. P. L.; ARKCOLL, D. B. Composição química do mesocarpo e do óleo de três populações de pupunha (Bactris gasipaes) do Rio Solimões, Amazonas, Brasil. Revista
Brasileira de Fruticultura, Jaboticabal, v. 20, n. 1, p. 115-118, 1998.

FERREIRA, C. D.; PENA, R. S. Comportamento higroscópico da farinha de pupunha (Bactris gasipaes). Ciência e Tecnologia de Alimentos, Campinas, v. 23, n. 2, p. 251-255, 2003.

GERMANI, R.; SILVA F. T. Fortificação de farinha de trigo e de milho com ácido fólico. Rio de Janeiro: Embrapa Agroindústria de Alimentos, 2004. (folheto).

GODOY, H. T.; RODRIGUEZ-AMAYA, D. B. Occurrence of cis-isomers of provitamin $A$ in Brazilian fruits. Journal of Agricultural and Food Chemistry, Washington, v. 42, n. 6 , p. 1306-1313, 1994.

GOIA, C. H. Processamento, caracterização e estabilidade da farinha de pupunha (Bactris gasipaes, H. B. K.). Manaus, 1992. 71 p. Dissertação (Mestrado em Tecnologia de Alimentos) - Instituto Nacional de Pesquisas da Amazônia - INPA.

GUY, R. Extrusión de los alimentos. Zaragoza: Acribia, 2001. $208 \mathrm{p}$

HORWITZ, W. Official methods of analysis. $16 \mathrm{ed}$. Washington: Association of Official Analytical Chemists, 1997.

MENEZES, E. W.; CARUSO, L.; LAJOLO, F. M. Avaliação da qualidade dos dados de fibra alimentar. Estudo em alimentos brasileiros. In: LAJOLO, F. M.; SAURA-CALIXTO, F.; PENNA, E. W.; MENEZES, E. W. (Eds.). Fibra dietética en Iberoamerica: tecnología y salud. 2 ed. São Paulo: Varela, 2001. p. 165-178. (cap. 11).

MORITA, A. S.; GOIS, V. A.; PRAÇA, E. F.; TAVARES, J. C.; ANDRADE, J. C; COSTA, F. B.; BARROS Jr., A. P.; SOUSA, A. H. Cristalização de melão pelo processo lento de açucaramento. Ciência Rural, Santa Maria, v. 35, n. 3, p. 705-708, 2005.

OLIVEIRA, A. N.; OLIVEIRA, L. A.; ANDRADE, J. S.; CHAGAS Jr., A. F. Produção de amilase por rizóbios, usando farinha de pupunha como substrato. Ciência e Tecnologia de Alimentos, Campinas, v. 27, n. 1, p. 61-66, 2007.

RICKARD, J. E.; BEHN, K. R. Evaluation of acid and enzyme hydrolitic methods for determination of cassava starch. Journal of Science of Food and Agriculture, Essex, v. 41, n. 4, p. 373-379, 1987

STATISTICAL ANALYSIS SYSTEM - SAS. SAS for Windows. Versão 8.0. Carry: Statistical Analysis System Institute, 1999.

SENTANIN, M. A.; AMAYA, D. B. R. Teores de carotenóides em mamão e pêssegos determinados por cromatografia líquida de alta eficiência. Ciência e Tecnologia de Alimentos, Campinas, v. 27, n. 1, p. 13-19, 2007

SOUZA, M. L. Processamento de cereais matinais extrusados de castanha-do-Brasil com mandioca. Campinas, 2003. 191 p. Tese (Doutorado em Tecnologia de Alimentos) - Universidade Estadual de Campinas - UNICAMP. 
Produção de snacks de terceira geração por extrusão de misturas de farinhas de pupunha e mandioca

CARVALHO, A. V. et al.

SOUZA, J. M. L.; NEGREIROS, J. R. S.; ÁLVARES, V. S.; LEITE, F. M. N.; SOUZA, M. L.; REIS, F. S.; FELISBERTO, F. A. V. Variabilidade físico-química da farinha de mandioca. Ciência e Tecnologia de Alimentos, Campinas, v. 28, n. 4, p. 907-912, 2008.

STONE, H. S.; SIDEL, J. L. Sensory evaluation practies. 2 ed. San Diego: Academic Press, 1993. 338 p.

UBOLDI-EIROA, M. N. Atividade de água: influência sobre o desenvolvimento de microrganismos e métodos de determinação em alimentos. Boletim do ITAL, Campinas, v. 3, n. 18, p. 353-383, 1981

UNITED STATES DEPARTAMENT OF AGRICULTURE - USDA. Composition of foods. Washington: Agricultural Research Center Service, 1963. 190 p. (Agriculture handbook n. 8)
VANDERZANT, C.; SPLITTSTOESSER, D. F. Compendium of methods for the microbiological examination of food. 3 ed. Washington: American Public Health Association, 1992. $1219 \mathrm{p}$

YUYAMA, L. K. O.; FÁVARO, R. M. D.; YUYAMA, K.; VANNUCCHI, $H$. Bioavailability of vitamin A from peach palm (Bactris gasipaes H.B.K.) and mango (Mangifera indica L.) in rats. Nutrition Research, Tarrytown, v. 11, n. 9, p. 1167-1175, 1991.

YUYAMA, L. K. O.; COZZOLINO, S. M. F. Efeito da suplementação com pupunha como fonte de vitamina A em dieta: estudo em ratos. Revista de Saúde Pública, São Paulo, v. 30, n. 1, p. 61-66, 1996. 